\title{
Assessment of contractility in intact ventricular cardiomyocytes using the dimensionless 'Frank-Starling Gain' index
}

\author{
Christian Bollensdorff • Oleg Lookin • Peter Kohl
}

Received: 2 March 2011 /Revised: 28 March 2011 / Accepted: 28 March 2011 /Published online: 15 April 2011

(C) The Author(s) 2011. This article is published with open access at Springerlink.com

\begin{abstract}
This paper briefly recapitulates the Frank-Starling law of the heart, reviews approaches to establishing diastolic and systolic force-length behaviour in intact isolated cardiomyocytes, and introduces a dimensionless index called 'Frank-Starling Gain', calculated as the ratio of slopes of end-systolic and end-diastolic force-length relations. The benefits and limitations of this index are illustrated on the example of regional differences in Guinea pig intact ventricular cardiomyocyte mechanics. Potential applicability of the Frank-Starling Gain for the comparison of cell contractility changes upon stretch will be discussed in the context of intra- and inter-individual variability of cardiomyocyte properties.
\end{abstract}

Electronic supplementary material The online version of this article (doi:10.1007/s00424-011-0964-z) contains supplementary material, which is available to authorized users.

C. Bollensdorff $\cdot$ P. Kohl

Cardiac Biophysics and Systems Biology,

The National Heart and Lung Institute, Imperial College,

London, UK

C. Bollensdorff $\cdot$ P. Kohl

Cardiac Mechano-Electric Feedback Group,

Department of Physiology, Anatomy and Genetics,

Oxford University,

Oxford, UK

O. Lookin

Institute of Immunology and Physiology,

Ural Branch of Russian Academy of Sciences,

Yekaterinburg, Russia

C. Bollensdorff $(\bowtie)$

Imperial College, National Heart and Lung Institute,

Heart Science Centre,

Hill End Road,

Harefield UB9 6JH, UK

e-mail: c.bollensdorff@imperial.ac.uk
Keywords Heart - Stretch · Positive inotropic response Cardiac mechanics $\cdot$ Mechanosensitivity

\section{Introduction}

General context

Externally homogeneous pressure-volume behaviour of the cardiac ventricles arises out of regionally heterogeneous structure-function characteristics of the myocardium [27]. The sequences of electrical activation, tissue contraction, and relaxation require individual cardiomyocytes in the heart to function in unison, in spite of spatially and temporally differing stress-strain dynamics to which they are exposed. Single cells therefore must be capable of adjusting their contractile properties on a beat-by-beat basis to spatial gradients in stress-strain dynamics, which are further modulated by temporally varying pressure-volume behaviour of the ventricles. A key response in this context is the pre-load dependence of contractility, referred to as the Frank-Starling (FS) law of the heart.

The Frank-Starling law

At the end of the nineteenth and the beginning of the twentieth century, Otto Frank and Ernest Henry Starling [15, 46] made major experimental and conceptual contributions to the then already well-known phenomenon [56] that the output of an isolated heart correlates, within limits, to prior fluid loading. The FS law describes a key auto-regulatory response, which allows rapid adaptation of cardiac contractile ability to changes in circulatory demand. The underlying positive inotropic ${ }^{1}$

\footnotetext{
${ }^{1}$ From inos (Greek for 'fibre') and trepein (Greek for 'to turn' or 'to change'): changing fibre activity, here with 'fibre' referring to cardiac myocyte rather than myofibrils (the term predates experimental confirmation of FS-type behaviour at the level of myofilaments).
} 
response to stretch occurs even in the absence of control interventions external to the heart (neural, hormonal), and it can thus be studied in isolated myocardial preparations. Without this intracardiac feedback, procedures such as heart transplantation would probably not have worked (at least not in the way we know them now).

The FS response counteracts the physical constrains, described in the law of Young (1773-1829) and Laplace (1749-1827), which limit the efficacy of translating surface tension (e.g. muscle contractile force) into intra-cavitary pressure, when the radius of a cavity increases. In other words, as cavity volume rises, more wall tension must be generated to produce the same pressure inside the cavity. Such volume increases occur on a beat-by-beat basis in the heart during ventricular filling, and without the FS response, the ventricles would become unable to empty at less challenging pre-loads ${ }^{2}$ than is the case physiologically. In heart failure, for example, ventricles dilate to an extent where the FS 'biology' may no more be able to compensate for the limitations imposed by the Young-Laplace 'physics', giving rise to significantly reduced ejection fractions in patients with pathologically dilated cardiac chambers [38, 52], even though the FS response itself may be preserved in the myocardium of failing hearts [22]. Of note, medical interventions that reduce chamber volume in patients with severe heart failure, such as by implantation of passive or active ventricular assist devices, may allow the myocardium to recover and, at least partially, regain functionality. The most successful recovery results were reported using combination therapies (device plus pharmacological intervention; [4]); however, underlying mechanisms and broader applicability are still a subject of (at times controversial) discussion.

The interrelation between intra-ventricular volume and pressure can be visualised by plotting pressure-volume behaviour in passive and mechanically active tissues. The relation of the pressures needed to achieve various filling levels during diastole is referred to as the (passive) enddiastolic pressure-volume relation (EDPVR). The endsystolic pressure-volume relation (ESPVR), in turn, describes the states reached at the end of (active) contraction.

The slopes of EDPVR and ESPVR are frequently used as indices of myocardial stiffness and cardiac contractility, respectively. These are affected by environmental parameters, such as circulating hormones, drugs, ion milieu, to name but a few (so one really ought to refer to each of them

\footnotetext{
${ }^{2}$ Note that the terms pre-load and after-load refer to different physical entities. Pre-load describes a spatial property (e.g. chamber volume in the heart or cell [really sarcomere] length in isolated tissue), while after-load is a force (e.g. downstream vascular pressure against which a chamber empties, or passive force against which an isolated cell or muscle contracts).
}

in plural). Hence, EDPVR and ESPVR may change in response to experimental interventions in one and the same sample, and they may differ regionally in the same heart, as well as between hearts (intra- and inter-individual variability). Furthermore, the use of end-systolic force as a descriptor of contractility-i.e. the ability to actively generate force and/or shortening - is limited by the fact that the end-systolic force, by definition, is total force, whereas the contractile ability is characterised by the difference between total and passive force (see Fig. 1).

In terms of experimental model systems, the whole heart (ideally in situ) offers the pathophysiologically most relevant preparation for the study of cardiac mechanoelectrical function. However, its inherent complexity ('Young-Laplace' effects, multi-cellular interactions including contributions by non-cardiomyocytes, regionally differing stress-strain patterns, etc.) and the limited extent to which interventions can be graded and responses be observed has called for lower dimensionality approaches. These include isolated trabeculae and papillary muscles [1, $20,50]$, single cardiomyocytes [23,36, 48], or contractile filaments [49] to explore the mechanisms underlying the FS response. As an equivalent to the pressure-volume relations in whole heart research, lower spatial complexity models explore end-diastolic and end-systolic force-length relations (EDFLR and ESFLR, respectively; see dashed [blue] and dotted [red] curves in Fig. 1), essentially reducing a complex three-dimensional problem to a pseudo-onedimensional case.

In spite of more than a century of research, the mechanisms underlying the FS response are still not completely resolved. Proposed contributions range from inter-filament spacing $[16,25]$ to changes in myofilament $\mathrm{Ca}^{2+}$ sensitivity [35], enhanced by co-operativity at the levels of both crossbridge [40] and $\mathrm{Ca}^{2+}$ binding [30], and they include contributions by the giant protein titin $[17,47$, 51], whether linked to contractile lattice spacing [18] or not [29]. For recent reviews on the molecular mechanisms of the FS behaviour, see [14, 45].

Even less is known about regional differences in the FS response, such as characterised by ESFLR and EDFLR [9], and about their interplay with other cellular mechanosensitive mechanisms, such as mechanical modulation of sarcolemmal [28, 55] and non-sarcolemmal [3, 24] ion channels and transporters in intact cardiomyocytes. The latter set of questions in particular can be addressed using intact (i.e. un-skinned) cardiomyocytes.

Measurement of ESFLR/EDFLR in intact cardiomyocytes

Techniques used to apply axial stretch to intact isolated cardiomyocytes include distension of agarose gels with embedded cells [39] or strain application to single cells 


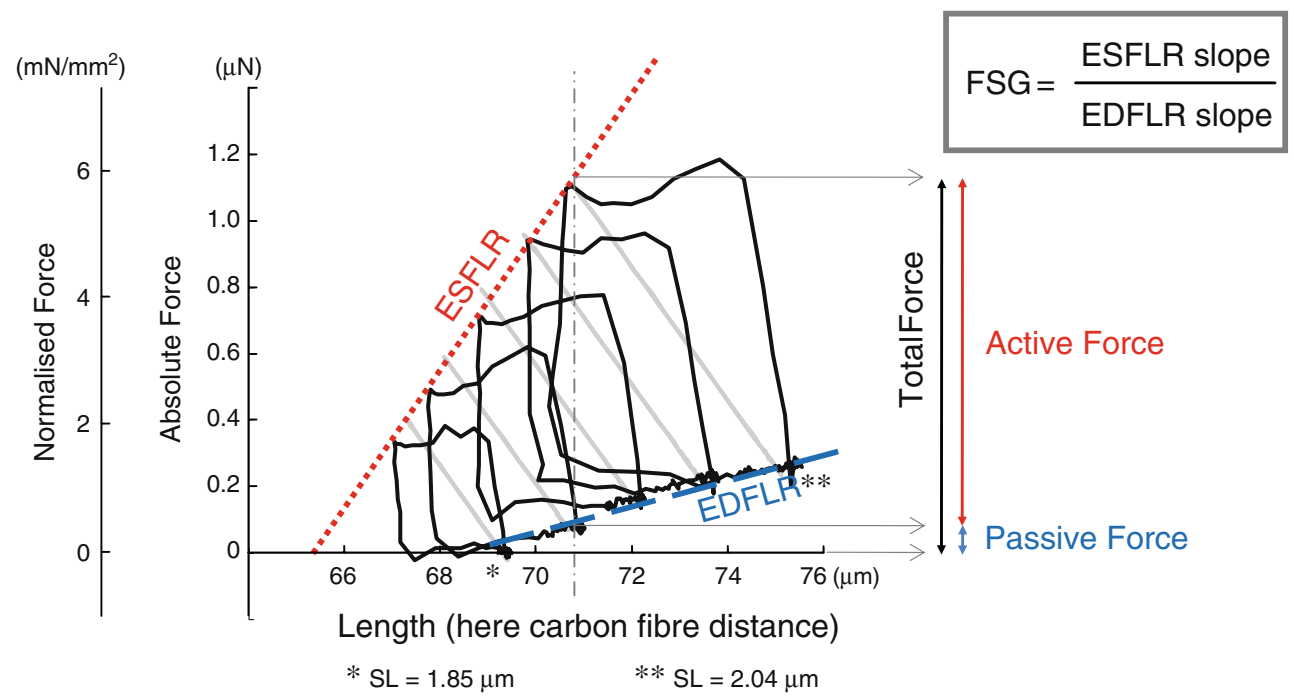

Fig. 1 Diagram representing end-diastolic and end-systolic forcelength relations (EDFLR and ESFLR, respectively) of a Guinea pig intact isolated ventricular cardiomyocyte, established using the carbon fibre (CF) technique. The EDFLR (dashed line, blue) is obtained by monitoring passive tension (force required to arrive at different pre-loads), while ESFLR (dotted line, red) is constructed by recording total force at the end of contractions, initiated from a range of different pre-loads (here covering a sarcomere length [SL] range from 1.85 to $2.05 \mu \mathrm{m}$ ). In Guinea pig, over the SL studied,

using suction pipettes [6], adhesives [5], or carbon fibres (CF) which can be attached to cells glue-free [31]. The CF technique in particular has been developed to a point where it can be used to prescribe to single cells a stress-strain environment that mimics relevant aspects of in situ dynamics, such as by exposing cells to work-loop-like contractions (see black traces in Fig. 1; see also [23, 36]).

For this technique, CF, mounted inside (much stiffer) glass capillaries, are lowered onto opposite ends of an isolated intact cell and, after attachment, used to lift the cell off the bottom of the perfusion chamber. Contraction is then monitored by tracking the movement of both CF tips. A variation of the technique employs $\mathrm{CF}$ of different stiffness, where the more rigid fibre serves as an anchor point, often left pressing the underlying cell fragment against the perfusion chamber, while CF bending is observed at one cell end only.

In both configurations, one can calculate active force generation, provided the compliance of moving $\mathrm{CF}$ is known (this has to be calibrated individually for each $\mathrm{CF}$, prior to use). If, in addition, the position of the CF-carrying glass capillary can be established (customarily by mounting the CF holder to a high-precision micromanipulator), it is possible to calculate $\mathrm{CF}$ bending even during externally imposed length changes, such as to determine passive force during diastole.

In this configuration, ESFLR (total force plotted as a function of end-systolic length; Fig. 1, black arrow) and
ESFLR is independent of the mode of contraction (e.g. auxotonic, grey lines; work-loop, black; data from [23]). Within this linear range of the Frank-Starling (FS) response, the slopes of ESFLR and EDFLR can be divided to yield a dimensionless index of the preload-dependent change in contractility - the Frank-Starling Gain (FSG). FSG calculation is independent of whether or not absolute or normalised force data are used (see scales on the left), and any errors introduced during normalisation of force to sample cross-section are cancelled out

EDFLR (passive force plotted as a function of end-diastolic length; Fig. 1, blue arrow) can be straightforwardly established for contractions initiated from a range of pre-loads. The difference between total and passive forces is the active force component (Fig. 1, red arrow), whose 'length-dependent recruitability' is at the heart of the FS law.

If the manipulator holding the CF is stationary, auxotonic contractions are observed (grey slanted lines; Fig. 1). Note that the 'ideal linear appearance' of auxotonic contractions is a consequence of establishing force as a function of spring deformation (CF tip movement); for a time-resolved trace of contraction and relaxation, both of which follow different dynamic trajectories, see Fig. 2b (experimental systems using quasi-isometric strain gauges tend to have the 'ideal linear part' in the isometric fragments of contractile activity). If motorised (e.g. using a Piezo-actuated platform to carry the CF holder), more complex and dynamic manipulations are possible, including isometric, isotonic, and work-loop (combined) contraction patterns (see black traces in Fig. 1). Interestingly, Iribe et al. report that over a preload range from 1.85 to $2.05 \mu \mathrm{m}$, ESFLR is unaffected by the mode of contraction in Guinea pig intact ventricular cardiomyocytes [23], simplifying the identification of EDFLR and ESFLR. This is also shown in Fig. 1, where auxotonic and work-loop contractions, initiated from various points on the EDFLR, arrive at the same points of the ESFLR. 

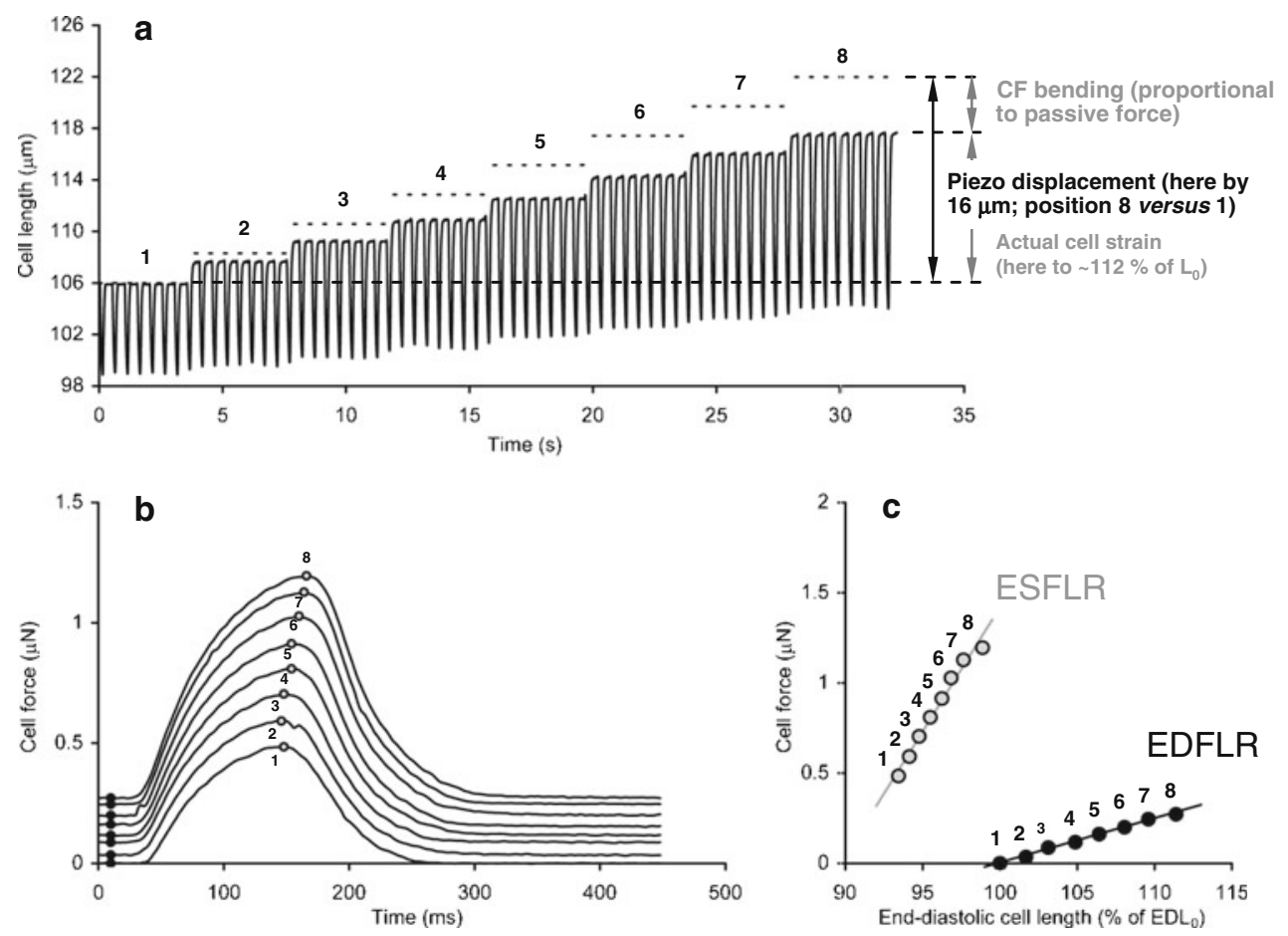

Fig. 2 Representative experimental records of a set of auxotonic twitches of Guinea pig ventricular cardiomyocyte, and extraction of ED/ESFLR using the CF technique. a Time series of cell shortening (pointing downwards) and consecutively applied increases in diastolic length (pointing upwards). Note that the piezo-motor command $(2-\mu \mathrm{m}$ step, indicated by dotted lines above respective curve segments) exceeds actual cell strain; the difference between

Studies of mechanical properties in intact ventricular cardiomyocytes have shown in several species (e.g. rat, Guinea pig, ferret) that sub-endocardial cells are significantly stiffer (steeper EDFLR) and show more effective pre-load-dependent force activation (steeper ESFLR) than sub-epicardial cells [9]. The physiological relevance of these differences may be related to the fact that subepicardial and sub-endocardial cells operate within different sarcomere length (SL) ranges in vivo. Indeed, in Guinea pig and rat, it has been reported that sub-epicardial myocytes have longer end-diastolic SL than sub-endocardial cells [7, 33, 41], while sub-endocardial cells show the greater percentage shortening during systole $(18 \%$ vs. $10 \%$, respectively; [12, 43]). Also, while stiffness is usually assessed as 'resistance to passive lengthening', one should not forget that (presumably the same) mechanical springs that prevent excessive distension of cardiac muscle cells also act to restore resting length after contraction [21]. In the context of greater relative shortening of sub-endocardial cells, their higher stiffness may convey a mechanical advantage for ventricular relaxation.

Steepness of EDFLR and ESFLR, both of which describe steady-state parameters, should not be confused with activation dynamics, where sub-epicardial layers of externally applied command and cell strain represents diastolic CF bending, used to calculate passive (diastolic) force. b Cell force recordings plotted as a function of time (force pointing upwards), superimposed for different pre-loads (numbers correspond to those in panels $\mathbf{a}$ and $\mathbf{c}$ ). $\mathbf{c}$ EDFLR and ESFLR (force plotted as a function of length, expressed as percentage of end-diastolic slack length, $\mathrm{EDL}_{0}$ ) using data points illustrated in panel $\mathbf{b}$

normal hearts across species show faster velocities of shortening and re-lengthening (or active force development and subsequent relaxation) than sub-endocardial layers [13, 53]. This behaviour has been related to transmural differences in myosin isoform expression [32]. In the context of electrical activation delays of sub-endocardial cells (compared to subendocardial ones), it gives rise to near-simultaneous peak force generation across the ventricular myocardium.

Interestingly, in situ, the earlier activation of subendocardial cell layers can give rise to a brief additional pre-strain of sub-epicardial cells at the onset of ventricular systole [2]. This endocardially induced epicardial strain may have a positive inotropic (i.e. FSlike) effect on the more compliant sub-epicardial cells, perhaps 'priming' them for the immanent contraction. However, direct observation of dynamic SL changes in native tissue has remained challenging, even for the optically more accessible sub-epicardial locations, because of motion-related imaging constraints [8]. This, together with attempts to develop micro-catheter-based confocal [42] and multi-photon [19] microscopy with a resolution that would potentially be suitable for in situ observation of sub-endocardial SL, forms interesting targets for further study. 


\section{The Frank-Starling Gain}

Presentation of passive and active mechanical properties using EDFLR/ESFLR (or EDPVR/ESPVR in the whole heart) is helpful in that they offer a summary description of behaviour over a range of pre- and after-loads. However, as mentioned before, force-length relations are affected by pathophysiologically relevant environmental properties, and they show significant intra- and inter-individual variability. Additional effects of experimental protocols, such as related to sample preparation and storage, increase variability and often make comparison difficult between species or between different preparations from the same species.

In addition, histo-anatomical differences must be accounted for when comparing absolute force (or pressure) values. Normalisation of force to cross-sectional area occupied by contractile tissue can be difficult. In multicellular samples, such as trabeculae or papillary muscles, the cross-sectional fraction not occupied by myocytes (e.g. capillaries, connective tissue) may vary by an order of magnitude or more. Thus, collagen content of trabeculae from normal rat hearts can range from $1 \%$ to nearly $100 \%$ and, over the sample diameter range commonly used in experiments, it varies between $2 \%$ and $20 \%$ [44]. This variability will be further enhanced when comparing normal to diseased tissue with different degrees of fibrosis.

For single-cell measurements, cross-sectional area is usually assessed using a formulaic approach based on measured cell 'width'. A common assumption is that cardiomyocyte cross section can be approximated by an ellipse, where the short-to-long axis ratio is about 1:3 [36]. Again, regional differences and disease conditions that affect this geometric ratio may introduce a systematic bias into normalised force calculations. Indeed, in a Guinea pig hypertrophy model, Wang et al. report an increased ventricular cardiomyocyte cross-sectional area (by 10$15 \%$ in some populations) in the absence of significant changes in cell width [54], highlighting the limitation of cell width measurements for the cross-sectional area estimation needed to normalise force recordings.

With these limitations in mind, and acknowledging that quantitative histo- or cytomorphological 3D reconstruction of samples is not usually feasible in routine research applications, it would seem helpful to describe the preload dependence of active force generation with a dimensionless index. This would aid comparison between preparations (e.g. across species, disease conditions, or developmental stages), as well as within (e.g. cells from different locations in the heart, responses to interventions). We therefore propose a simple index, called Frank-Starling Gain (FSG), which is defined as the ratio between the slopes of ESFLR and EDFLR (or ESPVR and EDPVR). The resulting index is a dimensionless descriptor of active force recruitability, i.e. of the positive inotropic response to stretch (Fig. 1).

Of course, this FSG index is not meant to replace EDFLR/ESFLR (EDPVR/ESPVR) or work-loop data presentations, where differences in off-set or the dynamic nature of contractions and their location relative to the origin of EDFLR/ESFLR (EDPVR/ESPVR) curves can be visualised. It should also be noted that the FSG is applicable, in the here proposed form, only to the linear portion of the force-length behaviour, i.e. outside nearmaximal length ranges.

At the same time, the FSG offers advantages beyond avoidance of sample cross-section-related errors, as it is focussed on length-dependent active force recruitment, capturing contributions from both ESFLR and EDFLR. This takes the FSG beyond the use of ESFLR (ESPVR) as a pre-load independent parameter, as the latter is used to characterise ventricular contractility on the basis of total force only (i.e. lumping active and passive force into one, rather than distinguishing between their potentially differential contributions to actual contractile ability). While the establishment of ESPVR as a clinically applicable descriptor of contractility offered advantages over hitherto employed approaches [26], variability in EDPVR can make significant contributions to the 'length-recruitable' mechanical work. This may range from increases in EDPVR slope during normal ageing [37] or in hypertrophy [11] to slope reductions that have been reported for disease conditions such as sepsis [10]. The combined effects of changes in end-systolic and enddiastolic mechanics are captured by the FSG.

\section{FSG application examples}

\section{Experimental approach}

For a detailed description, please see the on-line supplement. In short, ventricular cardiomyocytes were regionally isolated from Guinea pig hearts using enzymatic dissociation, yielding varying amounts of cells from up to six ventricular regions per isolation, including: right ventricular (RV) base (RVbase) and apex (RVapex), as well as sub-endocardial (endo) and sub-epicardial (epi) cells from matching regions in the left ventricle (LVbase_endo, LVbase_epi, LVapex_endo, LVapex_epi).

Cell pre-load was manipulated by exposure to passive axial stretch, applied using two CF attached to opposite cell ends, as described in detail elsewhere [23]. Cell length and width (averaged from three transversal measurements positioned near the centre and both cell ends) as well as SL were measured in the 'slack' resting state (i.e. without application of stretch), yielding $L_{0}, w_{0}$, and $\mathrm{SL}_{0}$, respectively. The distance between two CFs upon cell attachment 
was established as $\mathrm{EDL}_{0}$. Cells were then stretched by the application of 2- $\mu \mathrm{m}$ step increments (see Fig. 2a), applied during diastole with a constant velocity of $100 \mu \mathrm{m} \mathrm{s}^{-1}$. Note that actual cell length increments were less than the motor command steps applied, due to passive force causing diastolic CF bending. Cells were paced by field stimulation at $2 \mathrm{~Hz}$ and allowed to contract auxotonically. An example of auxotonic twitch force recordings at different pre-loads is shown in Fig. 2b. To exclude systematic errors caused by consecutively larger length increases, in confirmatory experiments, extensions of different amplitudes were applied in a random order, with return to $\mathrm{EDL}_{0}$ between runs (results from this protocol did not reveal principally different responses compared to the here illustrated consecutive stretch protocol).

The distance between CF (EDL) was measured optically, using the edge detection function of IonWizard (Ionoptix, Milton, USA), SL was established using a Fast Fourier Transformation-based algorithm in SarcLen (Ionoptix), and CF bending was calculated from the difference between piezo-manipulated CF holder position (PI Instruments, Karlsruhe, Germany) and optically tracked CF tip displacement.

Forces were calculated from $\mathrm{CF}$ bending, multiplied with a stiffness coefficient $\left(k ;\left[\mu \mathrm{N} \mu \mathrm{m}^{1}\right]\right)$ that was established by prior calibration of each $\mathrm{CF}$. For any $\mathrm{CF}$ pair, the effective stiffness coefficient was assumed to be the average of individual coefficients of the two CFs. Passive (enddiastolic) and total (end-systolic) force were calculated for each pre-load level from the cell shortening trace (Fig. 2a), averaged over at least five twitches after application of stretch. EDFLR and ESFLR were obtained from this set of the averaged twitches at all pre-loads studied (Fig. 2c). Note that end-systolic force was calculated for physiological (auxotonic) contractions; it is therefore smaller than it would be in isometric mode. However, over the length range studied, we assume that slopes of EDFLR and ESFLR are independent of the mode of contraction, as shown by Iribe et al. [23].

Data were evaluated using analysis of co-variances and Student's $t$ test; $P<0.05$ was considered as indication of significant differences between means. Data are presented as mean $\pm \mathrm{SEM}$ (for extended parameter listings, see OnLine Supplement).

Regional differences in cell geometry

A comparison of cell lengths and widths is provided in Table 1 . These data show that RV cells are significantly narrower (and thus presumed to have a smaller cross-sectional area), which will reduce their absolute force-generating ability. However, they are generally longer than LV cells, increasing their total length change potential. There was no significant difference between geometric parameters for RV cells isolated from basal and apical locations. Similarly, LV cell slack lengths and widths did not generally differ regionally, with the exception of LVapex_endo cells, which had a significantly higher slack length than LVbase_epi $(P<0.05)$. Ventricular cells isolated from different regions show similar SL in resting conditions $\left(\mathrm{SL}_{0}, 1.88-1.89 \mu \mathrm{m}\right)$, and LVapex_endo was again the only sub-population where $\mathrm{SL}_{0}$ differed from other LV regions: in spite of their overall larger total cell length, SL $(1.86 \pm 0.01 \mu \mathrm{m})$ was significantly smaller than elsewhere in the LV.

Table 1 Overview of cell geometry data, obtained at slack length for cells isolated from different regions of Guinea pig ventricle

\begin{tabular}{|c|c|c|c|}
\hline Region & Cell slack length (end-to-end) $[\mu \mathrm{m}]$ & $\mathrm{SL}_{0}[\mu \mathrm{m}]$ & Cell width $[\mu \mathrm{m}]$ \\
\hline RVbase & $146.6 \pm 3.3(46)^{\mathrm{a}}$ & $1.89 \pm 0.01(20)^{\mathrm{b}}$ & $18.6 \pm 0.7(53)^{\mathrm{c}}$ \\
\hline RVapex & $148.2 \pm 4.3(43)^{\mathrm{a}}$ & $1.89 \pm 0.01(15)^{\mathrm{b}}$ & $19.2 \pm 0.7(53)^{\mathrm{c}}$ \\
\hline RV (all) & $147.4 \pm 2.7(89)^{\mathrm{d}}$ & $1.89 \pm 0.01(35)$ & $18.9 \pm 0.5(106)^{\mathrm{d}}$ \\
\hline LVbase_epi & $136.1 \pm 4.3(27)$ & $1.88 \pm 0.01(20)^{\mathrm{e}}$ & $22.0 \pm 0.9(41)^{\mathrm{c}}$ \\
\hline LVbase_endo & $129.5 \pm 3.3(42)^{\mathrm{a}}$ & $1.88 \pm 0.01(26)^{\mathrm{e}}$ & $22.5 \pm 0.7(53)^{\mathrm{c}}$ \\
\hline LVapex_epi & $139.6 \pm 4.8(21)$ & $1.89 \pm 0.01(18)^{\mathrm{e}}$ & $21.7 \pm 0.8(28)^{\mathrm{c}}$ \\
\hline LVapex_endo & $147.7 \pm 4.4(28)^{\mathrm{a}}$ & $1.86 \pm 0.01(19)^{\mathrm{b}, \mathrm{e}}$ & $22.9 \pm 0.9(37)^{\mathrm{c}}$ \\
\hline LV (all) & $137.1 \pm 2.1(118)^{\mathrm{d}}$ & $1.88 \pm 0.00(83)$ & $22.3 \pm 0.4(159)^{\mathrm{d}}$ \\
\hline
\end{tabular}

Data shown as mean $\pm \mathrm{SEM}$, number of measurements in round brackets

Two-tailed unpaired $t$ test, $P<0.05$ :

${ }^{\text {a }}$ Significant difference between LVbase_endo and each of (RVbase; RVapex; LVapex_endo)

${ }^{\mathrm{b}}$ Significant difference between LVapex_endo and each of (RVbase; RVapex)

${ }^{\mathrm{c}}$ Significant difference between any of (LVbase_epi; LVbase_endo; LVapex_epi, LVapex_endo) and each of (RVbase; RVapex)

${ }^{\mathrm{d}}$ Significant difference between pooled LV (all) and RV (all)

${ }^{\text {e }}$ Significant difference between LVapex_endo and each of (LVbase_epi; LVbase_endo; LVapex_epi) 
Table 2 Slopes of ESFLR and EDFLR, and value of FSG index, for each tissue region studied

\begin{tabular}{|c|c|c|c|}
\hline Region & ESFLR slope $\left[\mathrm{mN} / \mathrm{mm}^{2}\right] /\left[\mathrm{ESL} / \mathrm{EDL}_{0}\right]$ & EDFLR slope $\left[\mathrm{mN} / \mathrm{mm}^{2}\right] /\left[\mathrm{EDL} / \mathrm{EDL}_{0}\right]$ & FSG [dimensionless] \\
\hline RVbase & $1.04 \pm 0.21(11)$ & $0.27 \pm 0.06(11)$ & $4.37 \pm 0.53(11)^{\mathrm{a}}$ \\
\hline RVapex & $1.01 \pm 0.13(14)^{\mathrm{b}}$ & $0.35 \pm 0.04(14)^{\mathrm{c}}$ & $2.99 \pm 0.31(14)^{\mathrm{a}}$ \\
\hline RV (all) & $1.02 \pm 0.12(25)^{\mathrm{d}}$ & $0.31 \pm 0.04$ & $3.59 \pm 0.32(25)^{\mathrm{d}}$ \\
\hline LVbase_epi & $0.72 \pm 0.09$ & $0.28 \pm 0.03(15)^{\mathrm{c}}$ & $2.66 \pm 0.19(15)^{\mathrm{a}}$ \\
\hline LVbase_endo & $0.80 \pm 0.10$ & $0.28 \pm 0.03(17)$ & $2.85 \pm 0.22(17)^{\mathrm{a}}$ \\
\hline LVapex_epi & $0.85 \pm 0.12(16)$ & $0.28 \pm 0.03(16)^{\mathrm{c}}$ & $3.08 \pm 0.25(16)^{\mathrm{a}}$ \\
\hline LVapex_endo & $0.68 \pm 0.10(11)^{\mathrm{b}}$ & $0.21 \pm 0.02(11)$ & $3.35 \pm 0.36(11)$ \\
\hline LV (all) & $0.79 \pm 0.05(59)^{\mathrm{d}}$ & $0.26 \pm 0.01(59)$ & $3.04 \pm 0.13(59)^{\mathrm{d}}$ \\
\hline
\end{tabular}

Data shown as mean $\pm \mathrm{SEM}$, number of measurements in round brackets

Unpaired $t$ test, $P<0.05$

${ }^{a}$ Significant difference between RVbase and each of (RVapex; LVbase_epi; LVbase_endo; LVapex_epi)

${ }^{\mathrm{b}}$ Significant difference between RVapex and LVapex_endo

${ }^{\mathrm{c}}$ Significant difference between LVapex_endo and each of (RVapex, LVbase_epi; LVapex_epi)

${ }^{\mathrm{d}}$ Significant difference between pooled RV (all) and LV (all)

\section{Regional differences in EDFLR, ESFLR and FSG}

ESFLR/EDFLR slopes and FSG, calculated for all investigated regions, are shown in Table 2. No significant intra-ventricular differences in ESFLR slopes were found (neither in LV nor RV). Inter-ventricular differences did exist: cells isolated from the RVapex had a higher ESFLR slope than cells from the LVapex_endo. The same held true when all cells, pooled from RV, were compared to all cell pooled from LV. The slope of EDFLR was significantly higher in RVapex cells compared to LV epicardial cells (both LVbase_epi and LVapex_epi). The FSG, in contrast, is highest in RVbase cells (4.37 \pm 0.53$)$, significantly exceeding all tissue regions except LVapex_endo. The same held true for the comparison of pooled RV cells with pooled LV, showing good general agreement with the

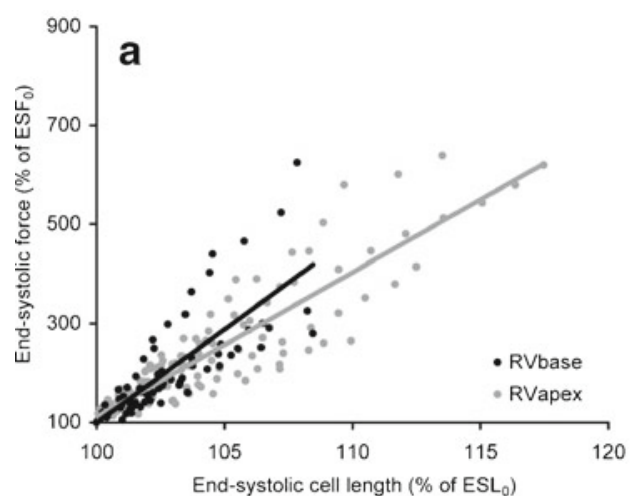

Fig. 3 Illustration of differences in pre-load recruitable contractile reserve in cells with similar ESFLR, identified by FSG. Cells from RVbase and RVapex yield similar ESFLR (a) and EDFLR (b) slopes (individual data points and best linear fit shown). The non-significant trends toward higher ESFLR and lower EDFLR normally prevailing focus on ESFLR. However, ESFLR alone may give false-negative information about recruitable contractile reserves, as EDFLR differences are not considered.

Example of potentially misleading ESFLR interpretation

Figure 3 demonstrates an example of potentially misleading interpretation of cardiomyocyte 'contractility', when assessed on the basis of ESFLR alone. For better visual representation, end-systolic force is shown as a percentage value of the active force generated by the cell at slack length. The slopes of neither ESFLR nor EDFLR (Fig. 3a, $\mathrm{b}$, respectively) differ in RV basal and apical cells (see Table 2). The assessment of 'contractility', based on endsystolic force only (even if one considered EDFLR as a

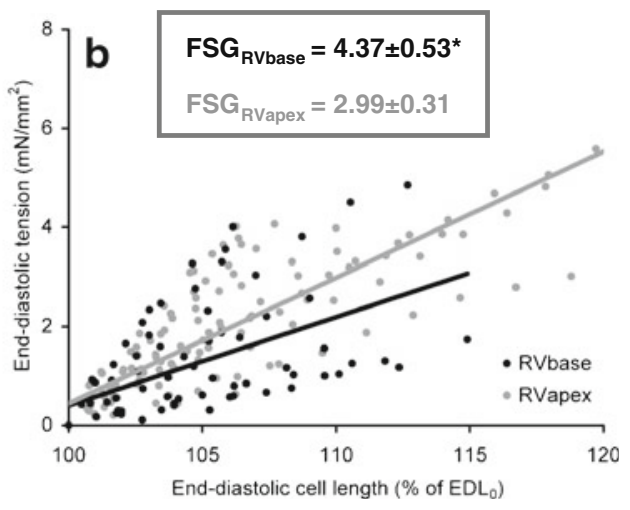

slopes in RVbase combine in a significantly increased FSG $\left({ }^{*} p<\right.$ 0.05 ), which is indicative of a higher contractile reserve compared to RVapex. End-systolic force is expressed as percentage of $\mathrm{ESF}_{0}$ and end-systolic length as percentage of $\mathrm{ESL}_{0}$ (see Table 2 for details) 
relevant but separate parameter) would result in the assumption that stretch-induced inotropy is not different in these cell populations. In contrast, the FSG index, calculated as the ratio of ESFLR and EDFLR slopes, shows a significantly higher recruitable contractile reserve in RVbase cells compared to RVapex.

\section{Discussion}

As new (potential) contributors to the mechanisms underlying the FS law of the heart continue to be uncovered, a full picture of how this physiologically relevant response is brought about has yet to emerge. In this context, it has not been easy to interrelate experimental findings, obtained at different levels of structural complexity, from filaments to skinned and intact cells, multi-cellular preparations and whole organ, even though the pre-load dependence of cardiac force generation can be observed in all of them. Even the comparison of insight from studies at the same level is not straightforward, as differences in experimental conditions, sample preparation, protocols, or observed parameters can influence the overall picture. Room for improvement is also present in the identification and use of clinically relevant contractility descriptors and their application to characterising responses to pharmacological or device-based interventions.

The here proposed FSG index may serve as a helpful extension to presently available tools. Being a dimensionless and 'active cross-section independent' parameter, it is less sensitive to errors in force normalisation. The FSG may be useful, therefore, for comparing responses to interventions within a preparation, between preparations from the same sample, between samples, or across model systems and even species. The underlying general approach (ratio of end-systolic to end-diastolic behaviour) can be applied in principle to various parameter types (e.g. length dependence of tension, volume dependence of pressure), and it would seem interesting to explore the suitability of this concept for experimental settings from filaments [49] to heterogeneous cardiac muscle duplexes [34] and whole organ [37].

An additional advantage of the FSG is that it takes into account the combined effects of the environment on both passive and active mechanical properties of the myocardium, as it is focussed on characterisation of active, rather than total, force responses to stretch. Thus, a positive lusitropic drug action (intervention reducing diastolic stiffness) could be highly relevant for cardiac performance even in the absence of changes in total (end-systolic) force generation (e.g. aiding faster and/or more complete ventricular filling). Such drug effect would be detected by the FSG, but not by descriptors of end-systolic behaviour only. After all, both end-systolic and end-diastolic length depend- ences are relevant for pre-load recruitable active contractile work, which is the essence of the FS law's relevance.

At the same time, the FSG index has several shortcomings. It does not convey absolute values of mechanical activity (so a weakly contractile heart, for example in sepsis, may still show a large change in pre-load recruitable relative contractile activity; [10]), and it does not convey where on the accessible pre-load range a preparation may operate at any given point in time. Also, interventionrelated shifts (say to larger or smaller pre-loads, which would be energetically relevant) will be missed. It is therefore important to consider FSG as an additional tool, which may be of utility in quantification of positive inotropic potential, focussing on active rather than total force effects.

Thus, the FSG is proposed as an additional description, not a replacement, for established approaches to characterising the FS response and cardiac contractility. Its ultimate utility will need to be assessed by application to a broader range of myocardial model systems.

Acknowledgment Supported by the British Heart Foundation.

Open Access This article is distributed under the terms of the Creative Commons Attribution Noncommercial License which permits any noncommercial use, distribution, and reproduction in any medium, provided the original author(s) and source are credited.

\section{References}

1. Allen DG, Kentish JC (1985) The cellular basis of the lengthtension relation in cardiac muscle. J Mol Cell Cardiol 17(9):821840

2. Ashikaga H, Van der Spoel TI, Coppola BA, Omens JH (2009) Transmural myocardial mechanics during isovolumic contraction. JACC Cardiovasc Imaging 2(2):202-211

3. Belmonte S, Morad M (2008) Shear fluid-induced $\mathrm{Ca}^{2+}$ release and the role of mitochondria in rat cardiac myocytes. Ann NY Acad Sci 1123:58-63

4. Birks EJ, George RS, Hedger M, Bahrami T, Wilton P, Bowles CT, Webb C, Bougard R, Amrani M, Yacoub MH, Dreyfus G, Khaghani A (2011) Reversal of severe heart failure with a continuous-flow left ventricular assist device and pharmacological therapy: a prospective study. Circulation 123(4):381-390

5. Bluhm WF, McCulloch AD, Lew WY (1995) Active force in rabbit ventricular myocytes. J Biomech 28(9):1119-1122

6. Brady AJ, Tan ST, Ricchiuti NV (1979) Contractile force measured in unskinned isolated adult rat heart fibres. Nature 282 (5740):728-729

7. Bryant SM, Shipsey SJ, Hart G (1997) Regional differences in electrical and mechanical properties of myocytes from guinea-pig hearts with mild left ventricular hypertrophy. Cardiovasc Res 35 (2):315-323

8. Bub G, Camelliti P, Bollensdorff C, Stuckey DJ, Picton G, Burton RA, Clarke K, Kohl P (2010) Measurement and analysis of sarcomere length in rat cardiomyocytes in situ and in vitro. Am J Physiol Heart Circ Physiol 298(5):H1616-H1625 
9. Cazorla O, Le Guennec JY, White E (2000) Length-tension relationships of sub-epicardial and sub-endocardial single ventricular myocytes from rat and ferret hearts. J Mol Cell Cardiol 32 (5):735-744

10. Cesar S, Potocnik N, Stare V (2003) Left ventricular end-diastolic pressure-volume relationship in septic rats with open thorax. Comp Med 53(5):493-497

11. Cingolani OH, Yang XP, Cavasin MA, Carretero OA (2003) Increased systolic performance with diastolic dysfunction in adult spontaneously hypertensive rats. Hypertension 41(2):249-254

12. Clark NR, Reichek N, Bergey P, Hoffman EA, Brownson D, Palmon L, Axel L (1991) Circumferential myocardial shortening in the normal human left ventricle. Assessment by magnetic resonance imaging using spatial modulation of magnetization. Circulation 84(1):67-74

13. Cordeiro JM, Greene L, Heilmann C, Antzelevitch D, Antzelevitch C (2004) Transmural heterogeneity of calcium activity and mechanical function in the canine left ventricle. Am J Physiol Heart Circ Physiol 286(4):H1471-H1479

14. De Tombe PP, Mateja RD, Tachampa K, Mou YA, Farman GP, Irving TC (2010) Myofilament length dependent activation. J Mol Cell Cardiol 48(5):851-858

15. Frank O (1899) Die Grundform des arteriellen Pulses. Z Biol 37:483526

16. Fuchs F, Wang YP (1996) Sarcomere length versus interfilament spacing as determinants of cardiac myofilament $\mathrm{Ca}^{2+}$ sensitivity and $\mathrm{Ca}^{2+}$ binding. J Mol Cell Cardiol 28(7):1375-1383

17. Fukuda N, Granzier HL (2005) Titin/connectin-based modulation of the Frank-Starling mechanism of the heart. J Muscle Res Cell Motil 26(6-8):319-323

18. Fukuda $\mathrm{N}, \mathrm{Wu} \mathrm{Y}$, Farman G, Irving TC, Granzier H (2005) Titinbased modulation of active tension and interfilament lattice spacing in skinned rat cardiac muscle. Pflugers Arch 449 (5):449-457

19. Gobel W, Kerr JN, Nimmerjahn A, Helmchen F (2004) Miniaturized two-photon microscope based on a flexible coherent fiber bundle and a gradient-index lens objective. Opt Lett 29(21):2521-2523

20. Guccione JM, Le Prell GS, De Tombe PP, Hunter WC (1997) Measurements of active myocardial tension under a wide range of physiological loading conditions. J Biomech 30(2):189-192

21. Helmes M, Trombitas K, Granzier H (1996) Titin develops restoring force in rat cardiac myocytes. Circ Res 79(3):619-626

22. Holubarsch C, Ruf T, Goldstein DJ, Ashton RC, Nickl W, Pieske B, Pioch K, Ludemann J, Wiesner S, Hasenfuss G, Posival H, Just H, Burkhoff D (1996) Existence of the Frank-Starling mechanism in the failing human heart. Investigations on the organ, tissue, and sarcomere levels. Circulation 94(4):683-689

23. Iribe G, Helmes M, Kohl P (2007) Force-length relations in isolated intact cardiomyocytes subjected to dynamic changes in mechanical load. Am J Physiol Heart Circ Physiol 292(3):H1487-H1497

24. Iribe G, Ward CW, Camelliti P, Bollensdorff C, Mason F, Burton RA, Garny A, Morphew MK, Hoenger A, Lederer WJ, Kohl P (2009) Axial stretch of rat single ventricular cardiomyocytes causes an acute and transient increase in $\mathrm{Ca}^{2+}$ spark rate. Circ Res 104(6):787-795

25. Irving TC, Konhilas J, Perry D, Fischetti R, De Tombe PP (2000) Myofilament lattice spacing as a function of sarcomere length in isolated rat myocardium. Am J Physiol Heart Circ Physiol 279(5): $\mathrm{H} 2568-\mathrm{H} 2573$

26. Kass DA, Maughan WL (1988) From 'Emax' to pressure-volume relations: a broader view. Circulation 77(6):1203-1212

27. Katz AM, Katz PB (1989) Homogeneity out of heterogeneity. Circulation 79(3):712-717

28. Kohl P, Bollensdorff C, Garny A (2006) Effects of mechanosensitive ion channels on ventricular electrophysiology: experimental and theoretical models. Exp Physiol 91(2):307-321
29. Konhilas JP, Irving TC, De Tombe PP (2002) Myofilament calcium sensitivity in skinned rat cardiac trabeculae: role of interfilament spacing. Circ Res 90(1):59-65

30. Kreutziger KL, Gillis TE, Davis JP, Tikunova SB, Regnier M (2007) Influence of enhanced troponin $\mathrm{C} \mathrm{Ca}^{2+}$-binding affinity on cooperative thin filament activation in rabbit skeletal muscle. J Physiol 583(Pt 1):337-350

31. Le Guennec JY, Peineau N, Argibay JA, Mongo KG, Garnier D (1990) A new method of attachment of isolated mammalian ventricular myocytes for tension recording: length dependence of passive and active tension. J Mol Cell Cardiol 22(10):1083-1093

32. Litten RZ, Martin BJ, Buchthal RH, Nagai R, Low RB, Alpert NR (1985) Heterogeneity of myosin isozyme content of rabbit heart. Circ Res 57(3):406-414

33. MacKenna DA, Omens JH, Covell JW (1996) Left ventricular perimysial collagen fibers uncoil rather than stretch during diastolic filling. Basic Res Cardiol 91(2):111-122

34. Markhasin VS, Solovyova O, Katsnelson LB, Protsenko Y, Kohl P, Noble D (2003) Mechano-electric interactions in heterogeneous myocardium: development of fundamental experimental and theoretical models. Prog Biophys Mol Biol 82 (1-3):207-220

35. McDonald KS, Moss RL (1995) Osmotic compression of single cardiac myocytes eliminates the reduction in $\mathrm{Ca}^{2+}$ sensitivity of tension at short sarcomere length. Circ Res 77(1):199-205

36. Nishimura S, Yasuda S, Katoh M, Yamada KP, Yamashita H, Saeki Y, Sunagawa K, Nagai R, Hisada T, Sugiura S (2004) Single cell mechanics of rat cardiomyocytes under isometric, unloaded, and physiologically loaded conditions. Am J Physiol Heart Circ Physiol 287(1):H196-H202

37. Pacher P, Mabley JG, Liaudet L, Evgenov OV, Marton A, Hasko G, Kollai M, Szabo C (2004) Left ventricular pressure-volume relationship in a rat model of advanced aging-associated heart failure. Am J Physiol Heart Circ Physiol 287(5):H2132-H2137

38. Petretta M, Vicario ML, Spinelli L, Ferro A, Cuocolo A, Condorelli M, Bonaduce D (2002) Combined effect of the forcefrequency and length-tension mechanisms on left ventricular function in patients with dilated cardiomyopathy. Eur J Heart Fail 4(6):727-735

39. Petroff MG, Kim SH, Pepe S, Dessy C, Marban E, Balligand JL, Sollott SJ (2001) Endogenous nitric oxide mechanisms mediate the stretch dependence of $\mathrm{Ca}^{2+}$ release in cardiomyocytes. Nat Cell Biol 3(10):867-873

40. Regnier M, Rivera AJ, Wang CK, Bates MA, Chase PB, Gordon AM (2002) Thin filament near-neighbour regulatory unit interactions affect rabbit skeletal muscle steady-state force-Ca ${ }^{2+}$ relations. J Physiol 540(Pt 2):485-497

41. Rodriguez EK, Omens JH, Waldman LK, McCulloch AD (1993) Effect of residual stress on transmural sarcomere length distributions in rat left ventricle. Am J Physiol 264(4 Pt 2):H1048-H1056

42. Rouse AR, Kano A, Udovich JA, Kroto SM, Gmitro AF (2004) Design and demonstration of a miniature catheter for a confocal microendoscope. Appl Opt 43(31):5763-5771

43. Sabbah HN, Marzilli M, Stein PD (1981) The relative role of subendocardium and subepicardium in left ventricular mechanics. Am J Physiol Heart Circ Physiol 240(6):H920-H926

44. Sands G, Goo S, Gerneke D, LeGrice I, Loiselle D (2011) The collagenous microstructure of cardiac ventricular trabeculae carneae. J Struct Biol 173(1):110-116

45. Shiels HA, White E (2008) The Frank-Starling mechanism in vertebrate cardiac myocytes. J Exp Biol 211(Pt 13):2005-2013

46. Starling EH (1918) The Linacre lecture on the law of the heart given at Cambridge 1915. Nature 101:43

47. Stuyvers BD, Miura M, Jin JP, Ter Keurs HEDJ (1998) $\mathrm{Ca}^{2+}$. dependence of diastolic properties of cardiac sarcomeres: involvement of titin. Prog Biophys Mol Biol 69(2-3):425-443 
48. Sugiura S, Nishimura S, Yasuda S, Hosoya Y, Katoh K (2006) Carbon fiber technique for the investigation of single-cell mechanics in intact cardiac myocytes. Nat Protoc 1(3):1453-1457

49. Suzuki M, Fujita H, Ishiwata S (2005) A new muscle contractile system composed of a thick filament lattice and a single actin filament. Biophys J 89(1):321-328

50. Ter Keurs HE, Rijnsburger WH, Van Heuningen R, Nagelsmit MJ (1980) Tension development and sarcomere length in rat cardiac trabeculae. Evidence of length-dependent activation. Circ Res 46 (5):703-714

51. Terui T, Sodnomtseren M, Matsuba D, Udaka J, Ishiwata S, Ohtsuki I, Kurihara S, Fukuda N (2008) Troponin and titin coordinately regulate length-dependent activation in skinned porcine ventricular muscle. J Gen Physiol 131(3):275-283

52. Vahl CF, Timek T, Bonz A, Kochsiek N, Fuchs H, Schaffer L, Rosenberg M, Dillmann R, Hagl S (1997) Myocardial length- force relationship in end stage dilated cardiomyopathy and normal human myocardium: analysis of intact and skinned left ventricular trabeculae obtained during 11 heart transplantations. Basic Res Cardiol 92(4):261-270

53. Wan X, Bryant SM, Hart G (2003) A topographical study of mechanical and electrical properties of single myocytes isolated from normal guinea-pig ventricular muscle. J Anat 202(6):525536

54. Wang X, Li F, Gerdes AM (1999) Chronic pressure overload cardiac hypertrophy and failure in guinea pigs: I. Regional hemodynamics and myocyte remodeling. J Mol Cell Cardiol 31 (2):307-317

55. White E (2006) Mechanosensitive channels: therapeutic targets in the myocardium? Curr Pharm Des 12(28):3645-3663

56. Zimmer HG (2002) Who discovered the Frank-Starling mechanism? News Physiol Sci 17:181-184 